\title{
Cytotoxin-Associated Gene-A - Positive Helicobacter pylori Strains Infection Increases the Risk of Recurrent Atherosclerotic Stroke
}

\author{
Marina Diomedi, ${ }^{*}+$ Paolo Stanzione, ${ }^{\dagger, \neq}$ Fabrizio Sallustio, ${ }^{\ddagger}$ Giorgia Leone, ${ }^{*}$ Antonio Renna, ${ }^{*}$ Giulia Misaggi, ${ }^{*}$ \\ Carla Fontana, ${ }^{\S}$ Patrizio Pasqualetti ${ }^{1, * *}$ and Antonio Pietroiusti ${ }^{\dagger+}$ \\ ${ }^{*}$ Clinical Neurology, Department of Neuroscience, Tor Vergata University, Rome, ${ }^{\dagger}$ IRCCS Clinica S. Lucia, Rome, ${ }^{\ddagger}$ Stroke Unit, Department of Neuroscience, \\ Tor Vergata University, Rome, §Department of Experimental Medicine and Biochemical Sciences, Tor Vergata University, Rome, "Medical Statistics \& \\ Information Technology, Fatebenefratelli Association for the Research, Isola Tiberina, Rome, ${ }^{* *}$ Casa di Cura San Raffaele Cassino e IRCCS San Raffaele \\ Pisana, Rome, ${ }^{\dagger+}$ Department of Biopathology, Tor Vergata University, Rome, Italy
}

\section{Keywords}

atherosclerosis, H. pylori, infections,

inflammation, recurrent stroke

Reprint requests to: Marina Diomedi, MD, Clinica Neurologica, Azienda Policlinico Tor Vergata, Viale Oxford, 81 - 00133 Roma, Italy. Tel.: +39-0620903119; Fax: +39-0620903118; E-mail: marina.diomedi@uniroma2.it

\begin{abstract}
Background: CagA-positive Helicobacter pylori infection has been found to be associated with a first-ever atherosclerotic stroke. The aim of this study was to investigate whether these strains represent an independent risk factor for recurrent atherosclerotic stroke.

Materials and Methods: We performed a longitudinal study of patients with a first-ever large vessels stroke and resulted positive at H. pylori serology. Patients had clinical examination 1 month after the acute event, and were subsequently visited or contacted by telephone up to 3 years at 6-month intervals. Sera obtained at the time of enrollment were frozen and analyzed for the presence of anti-CagA antibodies at the end of the study. The primary outcome event was any fatal or nonfatal stroke after the index stroke.

Results: One hundred seventy $H$. pylori-positive patients were included ( $\mathrm{n}=68$ CagA positive and $n=102$ CagA negative). No significant difference regarding age and other stroke risk factors was detected. According to Kaplan-Meier survival analysis, CagA-positive patients showed a significantly higher risk for stroke recurrence than CagA-negative ones $(45.6 \%$ vs $17.6 \% ; p<.001)$. Difference in the rate of recurrent stroke between the two groups persisted after Cox regression analysis taking into account possible confounding factors (hazard ratio $=3.5 ; 95 \% \mathrm{CI}=1.9-6.4 ; p<.001)$.

Conclusions: Infection with $H$. pylori CagA-positive strains increases the risk of recurrent atherosclerotic stroke. Seropositivity determination should be performed in order to identify high-risk patients requiring a strict clinical surveillance, and the possible beneficial effect of eradication therapy should be evaluated.
\end{abstract}

Stroke patients seem likely to be at high risk for recurrent stroke. The risk may vary with the type of cerebrovascular event, the highest one being observed in large artery stroke subtype [1,2]. For all types of cerebrovascular events, most recurrences are seen during the first weeks after stroke; thereafter, their rate decreases gradually [3-6]. By 5 years the cumulative risk of suffering a recurrent stroke is $25-30 \%[2,7,8]$.

Identification of modifiable risk factors is therefore strongly needed for a more effective prevention of recurrent episodes of cerebrovascular disease. Evidences indicate that inflammation contributes to both initiation and progression of atherosclerosis, and to acute rupture of atherosclerotic plaques, with superimposed thrombus formation increasing the risk of ischemic vascular events [9].

Several epidemiologic studies have reported an association between Helicobacter pylori bearing the cytotoxin-associated gene-A (CagA), a strong virulence factor, and ischemic stroke as a result of an atherosclerotic mechanism [10,11]. The findings of a longitudinal study, showing a strong predictive role of infection with CagA-positive strains for the incidence of first-ever stroke in patients with atherosclerosis suggested a causal relationship between the two 
events [12]. It is however, presently unknown whether patients with atherosclerotic stroke infected with these virulent strains carry a higher risk of relapsing.

In fact, these strains have been found to be associated with plaque destabilization, probably caused by the presence of a systemic inflammatory response induced by the organism [13].

Our primary goal was to prospectively examine the possible association between infection with CagA-positive strains and the risk of stroke recurrence during a 3-year follow-up in a population of patients with first-ever atherosclerotic stroke.

\section{Methods}

Nine hundred forty consecutive patients with acute first-ever ischemic stroke admitted at the Department of Neurology of Tor Vergata University of Rome during the years 2000 and 2003 were considered for the study. A standardized physical and neurological examination was carried out in all subjects. The main demographic and clinical data were collected at the time of enrolment of patients.

According to the criteria of the Trial of Org 10,172 in Acute Stroke Treatment (TOAST) investigators, patients were characterized into cardio-embolic, stroke as a result of large artery atherosclerosis, caused by small-artery occlusion (lacunar stroke), undefined stroke, or stroke from a rare cause [14]. The subtype definition was based on clinical features, vascular risk factors, and results of diagnostic studies (CT scan, MRI, 12-lead ECG, echocardiography).

In particular, all patients performed an ultrasonographic evaluation of neck and intracranial arterial vessels. Carotid arteries were assessed and defined by color flow B-mode Doppler ultrasound (Technos MP, Esaote Biomedica, Italy) according to the standardized criteria $[15,16]$. The vertebrobasilar system was evaluated as described by Bartels [17]. Intracranial vessels were examined by means of a Multidop T2 (DWL Elektronische Systeme GmbH, Sipplingen, Germany) and with color flow B-mode Doppler ultrasound (Technos MP, Esaote Biomedica, Italy). In some selected cases, in which the results of ultrasound examinations were not completely satisfactory, an MRI angiography was performed to confirm the presence of atherosclerotic lesions.

For the purpose of the present study, only patients bearing an occlusion or stenosis $\geq 50 \%$ of major brain arteries, fitting the criteria for stroke because of large vessel atherosclerosis were considered. Atherosclerotic lesions were characterized on the basis of the degree of lumen stenosis and surface features. Plaque was distincted as "stable" if the surface was smooth and "unstable" if the surface had ulcerations or height variations lower then $2 \mathrm{~mm}$. Measurement of IMT was performed on the common carotid arteries (CCAs), along $\sim 1.5 \mathrm{~cm}$ proximal to the flow divider and was defined as the mean of the maximum wall thickness for the near and far wall on the left and right side [18].

As far as traditional risk factors for large artery stroke are concerned, patients were defined as 1 , hypertensive if they had diastolic blood pressure $\geq 90 \mathrm{mmHg}$ and systolic blood pressure $\geq 140 \mathrm{mmHg}$ or if they had been treated for at least 1 year for this disorder; 2 , diabetic if they had fasting levels of glucose $\geq 126 \mathrm{mg} / \mathrm{dL}$ in two distinct instances or had been treated for at least 1 year with hypoglycemic drugs; 3 , active smoker if they reported a daily habit of $>10$ cigarettes per day for at least 1 year during the past 10 years; and 4, hypercholesterolemic if they had levels of total cholesterol $>220 \mathrm{mg} / \mathrm{dL}$ or had been treated for at least 1 year with lipid-lowering drugs. Body mass index (BMI; $\mathrm{kg} / \mathrm{m}^{2}$ ) was taken as a measure of obesity.

Exclusion criteria were: previous therapy aimed at eradication of H. pylori, history of peptic ulcer disease, evidence of connective tissue, neoplastic or hematologic diseases, and indication for early ( $<3$ months) carotid surgery or stenting.

Patients were treated according to the department's guidelines with antithrombotic and antihypertensive medication, and advice was given concerning lifestyle.

Informed written consent was obtained by patients or, when not possible, by their closest relatives. The ethics committee of our institution approved the study protocol.

\section{Serologic Data}

At the time of enrolment, a blood sample was obtained. A serum aliquot was immediately processed for the evaluation of the presence of immunoglobulin (Ig) G antibodies to H. pylori as inclusion criterion for the study, whereas the remaining serum was frozen at $-80{ }^{\circ} \mathrm{C}$. IgG against H. pylori were evaluated using ELISA (Pyloriset, ORION Diagnostica, Espoo, Finland). Titers $\geq 300$ units were regarded as positive. C-reactive protein (CRP) was assessed using rate nephelometry (Behring NA latex CRP, Behring Institute, Scoppito, l'Aquila, Italy) and, in samples with $<0.25 \mathrm{mg}$ of CRP/dL, using enzyme immunoassay (Imx, Abbott Laboratories, North Chicago, IL), calibrated with the World Health Organization's International Reference Standard for CRP Immunoassay. The range of value detected by the assay is 0.005 to $3 \mathrm{mg} / \mathrm{dL}$ [19]. The normal upper limit was considered $3 \mathrm{mg} / \mathrm{dL}$. Because serum levels of CRP may be strongly influenced by recent ischemic events [20,21], a further venous blood sample was obtained at 1 -month visit, for the evaluation of this parameter.

In patients testing $H$. pylori-positive frozen sera were examined for IgG antibodies to CagA protein detection in 
a blind fashion at the end of the study. Antibodies to CagA protein were investigated using ELISA (CTX, Eurospital, Trieste, Italy). The interassay variations for IgG to $H$. pylori and to CagA were not $>10 \%$.

Patients were asked to collect specimens from their first stools. For patients unable to do so, the procedure was performed by the nurses. Samples were stored at $-20{ }^{\circ} \mathrm{C}$ and analyzed concomitantly with serum antibodies to CagA until the time of serologic CagA determination. The specimens were analyzed for $H$. pylori antigens using HpSA immunoassay as described by its manufacturer. A commercial kit, Primier platinum HpSA (Meridian Diagnostic, Cincinati, Ohaio USA), was used.

\section{Outcomes}

Patients were asked to visit the outpatient clinic of our institution 1 month after the acute event and every 6 months for at least 36 months for cerebrovascular disease.

During each visit, a standardized clinical and neurological examination was performed, and history of recurrent stroke was considered to be reliable only when confirmed by hospital records. Diagnosis was determined according to the criteria of the National Institute of Neurological Disorders and Stroke for the classification of cerebrovascular events [22]. Recurrent stroke was defined as a new neurological deficit fitting the standard definition of a stroke, which occurred after a period of at least 24 hours of neurological stability [23]. Only events compatible with a large artery atherosclerotic mechanism were considered.

Therefore, we excluded any new deficit that occurred within 24 hours or that was thought to be attributable to edema, mass effect, or hemorrhagic transformation of the incident infarct. The interval period between recurrent stroke and index stroke was calculated in months.

Patients not presenting at the scheduled follow-up visit were contacted by telephone. In case of death, we ascribed it to a fatal stroke only if it was possible to obtain the pertinent documentation, or if death had occurred during hospitalization at our institution.

\section{Statistical Analyses}

The number of patients to be followed-up in order to assess the prognostic role of CagA was not planned a priori. However, the sample size obtained from all patients with acute first-ever ischemic stroke admitted at the Department of Neurology of Tor Vergata University of Rome during the year 2000 and 2003 can be considered appropriate for the following considerations. During the 3-year follow-up, 49 recurrence events occurred. Peduzzi et al. suggested that the event-per-variable ratio (EPV) for multivariable follow-up studies should be 10 [24,25]. Therefore, 50 events (approximately) would allow to reliably estimate the effect of 5 variables. In the present study, there were eight variables, one of interest (CagA) plus seven relevant covariates (age, gender, body mass index, smoking, diabetes, dyslipidemia, hypertension), resulting in an expected $\mathrm{EPV}=49 / 8$ corresponding to 6.12 . Because this is lower than 10, multivariable analysis has been performed also after a data reduction of covariates, namely collapsing all five risk factors (overweight, smoking, diabetes, dyslipidemia, hypertension) in a composite score, providing an $\mathrm{EPV}=49 / 4$ corresponding to 12.25 . Therefore, the sample size can be considered appropriate to identify the prognostic value of the potentially predictive factors.

In terms of effect size (of the variable of interest, i.e. CagA), a sample size of 170 patients provides a power of $80 \%$ to detect an odds ratio of 3 with bilateral alpha set at 0.05 , even in case of slight unbalancing between the two groups (up to $1.5: 1$ ). With respect to this issue, the present study could be underpowered for an odds ratio close to 2 or less.

Statistical analysis was performed with the appropriate software (sPss for windows version 13). Student's $t$-test and chi-square test were, respectively, used to compare continuous or categorical variables.

For survival analysis purpose, a 36-month follow up was chosen as the time to stop the study. The Kaplan-Meier method and the log-rank test (Mantel-Cox) were used to compare event-free survival among patients with and without H. pylori CagA-positive strains. With the use of proportional hazards analysis, hazard ratios (HR) and 95\% confidence intervals $(95 \% \mathrm{CI})$ were generated for the unadjusted association between CagA status or other baseline characteristics and the end point of fatal or nonfatal stroke. Hazard ratios were then adjusted for the confounding effects of other baseline characteristics, including age, sex, smoking status, BMI, and the presence or absence of diabetes mellitus, hypercholesterolemia, and hypertension (Cox regression analysis). In addition, the assumption of the hazard proportionality of the Cox model was checked and the appropriate adjustment performed to obtain more robust estimates of the effects. In particular, Cox regression with time-dependent covariates was applied.

We considered as "censored" in survival analysis all patients for whom the duration of the study was limited in time for different reasons such as undergoing to carotid thromboendoarterectomy, or carotid angioplasty, death from causes other than recurrent stroke, and drop out during the follow up period.

\section{Results}

Of 282 patients fitting the diagnostic criteria for atherosclerotic stroke, 170 were included in the study for 
being positive to the presence of IgG antibodies against H. pylori. Twenty-seven patients were excluded because of previous ischemic event, 9 because of programmed early surgical or percutaneous carotid treatment, 5 for previous H. pylori eradication therapy, 6 because affected by immune or neoplastic diseases, and 65 patients for testing negative to H. pylori.

One hundred-two $(60 \%)$ patients tested CagA negative and $68(40 \%)$ tested CagA positive. The clinical characteristics of H.pylori CagA-negative and H.pylori CagA-positive patients are shown in Table 1. There were no significant differences in traditional risk factors distribution between CagA-negative and -positive patients, except for the prevalence of hypercholesterolemia, which was detected with increased frequency among CagA-positive patients.

According to the finding of HpSA stool antigen test, 84 of 102 CagA negative $(85.7 \%)$ and 53 of 68 CagA positive $(77.9 \%)$ tested positive for active infection.

CagA-positive patients showed higher IMT values than those negative for CagA ( $1.05 \pm 0.13 \mathrm{~mm}$ vs $0.99 \pm 0.12 \mathrm{~mm}$; $p=.003)$. There was a significant association between CagA positivity and echographic pattern of plaque instability. In fact, $66.7 \%$ of patients with an unstable plaque were CagA positive in comparison to $23.5 \%$ with stable plaque $(p<.005)$.

Concerning medical treatment, all patients took antiplatelet drugs and all hypercholesterolemic patients took statins. Angiotensin-converting-enzyme inhibitors were used in $58.8 \%$ of patients in the CagA-positive group and in $63.7 \%$ of patients in the CagA-negative group; beta blockers in $33.8 \%$ and $31.4 \%$, respectively. Concerning CRP values, Mann-Whitney test for group comparison showed higher values in CagA-positive than in CagAnegative patients (median, 4.1; range, 1-9 vs median, 3.4; range, $0.9-8.1 ; p=.05)$.

One-hundred five patients had a significant extracranial vessel stenosis, 40 patients had a significant intracranial stenosis, and 25 patients had both extra- and intracranial stenosis.

The cumulative incidence of recurrent stroke in the total population was $28.8 \%$ (49 out of 170 ). It was however, much higher in CagA-positive patients ( 31 of $68,45.6 \%$ ), than in CagA-negative ones ( 18 of $102,17.6 \%$ ), a statistically significant difference (chi-square $=15.53$, d.f. $=1, p<.001$ ). Adjusting for the different length of follow-up until recurrence/censoring, the persons-year method allowed to estimate that the annual rate of recurrence was $8 \%$ in CagA negative and $25.5 \%$ in CagA positive. Kaplan-Meier survival curves of the two groups significantly differed (log rank test; $p<.001$ ) (Fig. 1).

Event-related mortality was observed in nine patients $(18.4 \%)$, its incidence being not significantly different between the two groups (6/31 CagA positive vs 3/18 CagA negative, $p=.183)$; similarly, no significant difference was found in global mortality $(9 / 68,13.2 \%$ in CagA positive vs $11 / 102,10.8 \%$ in CagA negative ones; $p=.627$ ).

Among survivors, 75 patients (23 CagA-positive and 52 CagA-negative patients) were free from recurrence at the end of the follow up; 35 patients were censored - 11 because underwent a delayed carotid angioplasty or endarterectomy, 6 because stroke recurrence was likely to the result of a cardioembolic origin, and 18 because were lost at various times during follow-up.

After verifying the effect of each variable (age, gender, BMI, smoking, diabetes, hypercholesterolemia, hypertension

Table 1 Demographic, clinical characteristics of patients and medical treatment during the follow up

\begin{tabular}{|c|c|c|c|}
\hline & $\begin{array}{l}\text { H. pylori CagA- } \\
(\mathrm{n}=102)\end{array}$ & $\begin{array}{l}\text { H. pylori CagA+ } \\
(\mathrm{n}=68)\end{array}$ & $p$ \\
\hline Age, y & $67.8(11.1)$ & $68.9(10.8)$ & .513 \\
\hline \multicolumn{4}{|l|}{ Mean (SD) } \\
\hline Male sex, n (\%) & $74(72.5 \%)$ & $47(69.1 \%)$ & .628 \\
\hline $\mathrm{BMI}, \mathrm{kg} / \mathrm{m}^{2}$ & $27.4(4.6)$ & $27.1(5.7)$ & .672 \\
\hline \multicolumn{4}{|l|}{ Mean (SD) } \\
\hline Current smokers, n (\%) & $38(37.3 \%)$ & 27 (39.7\%) & .747 \\
\hline Diabetes, $n(\%)$ & $29(28.4 \%)$ & $24(35.3 \%)$ & .344 \\
\hline Hypercholesterolemia, n (\%) & $33(32.4 \%)$ & $35(51.5 \%)$ & .013 \\
\hline Hypertension, $n(\%)$ & $70(68.6 \%)$ & $44(64.7 \%)$ & .594 \\
\hline $\mathrm{CRP}, \mathrm{mg} / \mathrm{dL}$ & $3.4(0.9-8.1)$ & $4.1(1-9)$ & .015 \\
\hline \multicolumn{4}{|l|}{ Median (min-max) } \\
\hline $\mathrm{IMT}, \mathrm{mm}$ & $0.99 \pm 0.12$ & $1.05 \pm 0.13$ & .003 \\
\hline Instable plaque (plaque instability), n (\%) & $26(25.5)$ & $52(76.5)$ & .000 \\
\hline
\end{tabular}

For continuous variables, $t$-test was used; in case of variance heterogeneity, degrees of freedom were adjusted. Gender and risk factors were analyzed with chi-square. Mann-Whitney test was applied to CRP values. BMI: body mass index; CRP: C-reactive protein. 


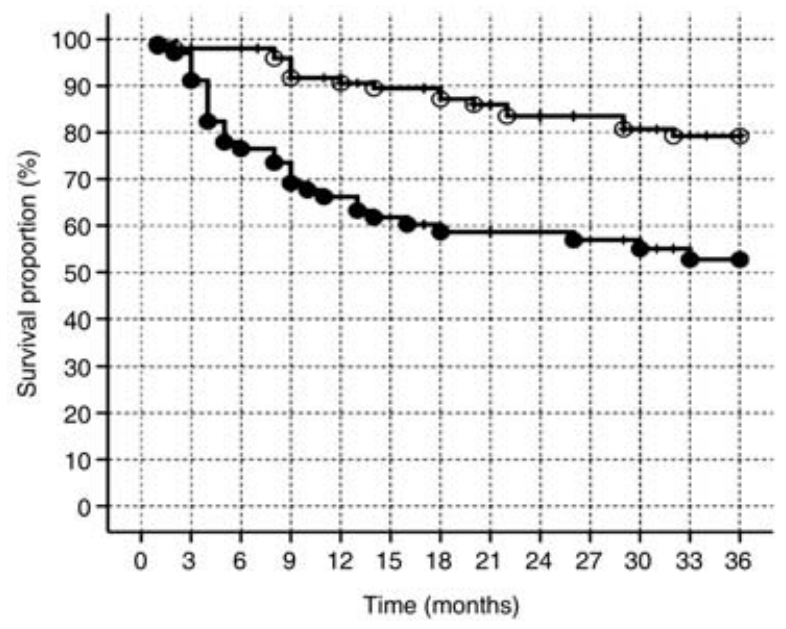

Figure 1 Kaplan-Meier curves for CagA-negative (open circles) and CagA positive (closed circles) patients. The considered event was the recurrence of a stroke after incident large artery ischemic stroke.

and CagA) separately on stroke recurrence (Table 2 singlevariable model), the first multiple Cox regression analysis was performed choosing a forward selection method (based on likelihood ratio). This procedure indicated that the only predictors of stroke recurrence were smoking $(\mathrm{HR}=2.92 ; 95 \% \mathrm{CI}=1.64-5.18 ; p<.001)$ and the presence of CagA-positive strains (HR $=3.35$; $95 \% \mathrm{CI}=1.87$ $6.02 ; p<.001)$. No other variables entered the model (consistently, $p>.20$ ). Because age could typically play an interactive effect, the interaction age*smoking and age ${ }^{\star}$ CagA positivity was verified but the result was not significant $(p>.20)$. The EPV ratio (see Statistical
Analysis section) was below the recommended level; thus, the risk factors were summed in a single score (ranging between 0 and 5), substituting the five single factors. The previous finding was confirmed $(p<.001$ for CagA positivity and $p=.024$ for risk factor score). However, the previously mentioned models did not completely satisfy the assumptions of the Cox model, the most relevant being the proportionality of hazards. Indeed, the diagnostic statistics indicated that there was a nonlinearity in the log-minus-log (LML) plot, confirmed by the interaction CagA positivity*time $(p=.056)$, and a dependence of the partials Schoenfeld residuals on time $(r=0.9, p<.001)$. For such reason, a Cox regression with time-dependent covariates was applied. This model did not change the relevance of the two factors but provided different estimates of the effects. More precisely, HR for CagA positivity resulted $7(95 \% \quad \mathrm{CI}=2.4-20.4 ; p<.001)$, whereas HR for smoking resulted 4.6 (95\% CI = 1.7-12.4; $p=.002)$.

\section{Discussion}

Recent investigations suggest a pathogenetic role of chronic infection with CagA-positive H.pylori strains in atherosclerotic stroke, as these strains have been found to be associated with increased intima media thickness (IMT) of the common carotid arteries and with a poorer stroke outcome measured at 1 month with National Institute of Health Stroke Scale (NIHSS) in patients with large vessel stroke, in which the atherosclerotic process represents the main pathophysiological mechanism [11].

In the present study we prospectively evaluated the possible influence of CagA seropositivity on the risk of atherosclerotic stroke recurrence.

Table 2 Cox regression analysis for stroke recurrence

\begin{tabular}{|c|c|c|c|c|c|c|c|c|c|c|c|c|}
\hline & \multicolumn{6}{|c|}{ Single-variable model } & \multicolumn{6}{|c|}{ Multivariable model } \\
\hline & \multirow[b]{2}{*}{ B } & \multirow[b]{2}{*}{ SE } & \multirow[b]{2}{*}{$p$} & \multirow[b]{2}{*}{$\mathrm{HR}$} & \multicolumn{2}{|l|}{$95 \% \mathrm{Cl}$} & \multirow[b]{2}{*}{ B } & \multirow[b]{2}{*}{ SE } & \multirow[b]{2}{*}{$p$} & \multirow[b]{2}{*}{$\mathrm{HR}$} & \multicolumn{2}{|l|}{$95 \% \mathrm{Cl}$} \\
\hline & & & & & lower & upper & & & & & lower & upper \\
\hline Age & 0.012 & 0.014 & .410 & 1.01 & 0.98 & 1.04 & & & & & & \\
\hline Male sex & 0.036 & 0.316 & .909 & 1.04 & 0.56 & 1.93 & & & & & & \\
\hline BMI & 0.019 & 0.030 & .530 & 1.02 & 0.96 & 1.08 & & & & & & \\
\hline Smoking & 0.966 & 0.291 & .001 & 2.63 & 1.49 & 4.65 & 1.071 & 0.293 & .000 & 2.92 & 1.64 & 5.18 \\
\hline Diabetes & 0.239 & 0.296 & .420 & 1.27 & 0.71 & 2.27 & & & & & & \\
\hline Hypercholesterolemia & 0.001 & 0.293 & .996 & 1.00 & 0.56 & 1.78 & & & & & & \\
\hline Hypertension & 0.284 & 0.324 & .379 & 1.33 & 0.71 & 2.51 & & & & & & \\
\hline CagA positivity & 1.115 & 0.297 & .000 & 3.05 & 1.70 & 5.45 & 1.209 & 0.299 & .000 & 3.35 & 1.87 & 6.02 \\
\hline
\end{tabular}

BMI: body mass index. 
In fact, we found a significant correlation between the presence of CagA antibodies and the incidence of recurrent stroke, although overall mortality and event-related mortality did not significantly differ between CagApositive and CagA-negative patients. This finding shed light on the interaction of infection with the natural history of atherosclerotic stroke. Indeed, several previous studies have shown that CagA-positive strains are associated with a first episode of large artery ischemic stroke [10]. However, from a pathophysiologic point of view, this relation might merely reflect the involvement of the infection in the very early stages of the atherosclerotic process, as shown by the positive relationship between infection with CagA-positive strains and increased intima/media thickness (IMT) of the common carotid arteries both in healthy subjects and in patients with atherosclerotic stroke [26]. Our findings suggest that the presence of infection may have a causal role even in the later stages of the disease. The exact mechanism underlying this association is not explained by the present study. However, one recent report showed that infection with CagA-positive strains may be linked with plaque instability [13], the final crucial event in the development of atherosclerotic stroke. Our data strongly support a causal effect of CagA-positive strains in this process in both first and recurrent episodes.

How CagA-positive strains may lead to recurrent plaque instability and thus to recurrent large artery ischemic stroke remains speculative.

This correlation might be the result of a deleterious local effect of infection and the consequent inflammation at the plaque level. In fact, some studies report the presence of antigenic material belonging to the organism at the plaque level: the local inflammatory reaction following the presence of the bacterium might induce the instability of the plaque $[27,28]$.

Our findings, showing the presence of active infection in the majority of the patients, give support to the inflammatory hypothesis.

Another possible mechanism may be linked to the fact that virulent $H$.pylori strains have common antigenic sequences with vascular wall components. The antibodies elicited by the infection may cross-react with these antigens, causing an immunomediated inflammatory reaction, leading to plaque instability. Interestingly, antigenic sequences recognized by anti-CagA antibodies are not exposed under normal conditions; however, they may be easily recognized by circulating antibodies, when the integrity of the arterial wall is lost, such as in atherosclerotic patients [29].

An additional pathogenetic mechanism might be linked to the activation of the host inflammatory response to the chronic infection of $H$. pylori virulent strains, independently from its presence in the atherosclerotic plaque. Our findings, showing increased baseline levels CRP (a sensitive marker of systemic inflammation) in patients with subsequent recurrence, suggest that systemic inflammation is probably involved in the process.

An independent causal role of smoking habit in the increased risk of stroke recurrence has been found in our patients. There is convincing evidence that cigarette smoking has numerous effects that may contribute to stroke risk promoting atherosclerosis through vascular inflammation and oxidative stress [30-32]. Therefore, in our population the infection with virulent $H$. pylori strains may amplify the unfavorable effect of smoking on endothelium. However, we cannot draw definitive conclusions about the role of active smoking on stroke recurrence as we have no data on the prevalence of smoke cessation after the inclusion in the study.

The main limitation of the present study is the relatively small size of our study group and therefore we have to be cautious about the predictive role of infection by H. pylori virulent strains; however, strict criteria were used to select atherosclerotic stroke patients and for diagnosing stroke recurrence.

Moreover, we have not taken in consideration the vascular district of the new event and the possible correlation with the previous, but we selected only patients with a recurrent stroke of an atherosclerotic origin.

A theoretical limitation of the study is the lack of a control population of $H$. pylori-negative patients. However, all previous studies on this subject invariably showed a higher prevalence of ischemic stroke in H.pylori-positive CagA negative patients in comparison to H.pylori negative patients $[10,11,33,34]$. Therefore, we feel that the inclusion of this population would not have substantially changed our findings.

A causal role has not been demonstrated but only presumed; however, our findings may have therapeutic implications. In fact, all strains of $H$. pylori may be eradicated by a short course of specific antibiotic therapy [35], and the level of antibody response tends to disappear with time after eradication [36]. Whether this therapeutic approach will be useful for the prevention of recurrent stroke of atherosclerotic origin may be answered by further clinical studies.

\section{References}

1 Petty GW, Brown RD, Whisnant JP, Sicks JD, O'Fallon WM, Wiebers DO. Ischemic stroke subtypes: a population-based study of functional outcome, survival, and recurrence. Stroke 2000;31:1062-8.

2 Modrego PJ, Mainar R, Turull L. Recurrence and survival after first-ever stroke in the area of Bajo Aragon, Spain. A prospective cohort study. J Neurol Sci 2004;224:49-55.

3 Lovett JK, Dennis MS, Sandercock PAG, Bamford J, Warlow CP, Rothwell PM. Very early risk of stroke after a transient ischaemic attack. Stroke 2003;34:e138-40. 
4 Bamford J, Sandercock P, Dennis M, Burn J, Warlow C. A prospective study of acute cerebrovascular disease in the community: the Oxfordshire Community Stroke Project, 1981-86. Incidence, case fatality rates and overall outcome at one year of cerebral infarction, primary intracerebral and subarachnoid haemorrhage. J Neurol Neurosurg Psychiatry 1990;53:16-22.

5 Johnston SC, Gress DR, Browner WS, Sidney S. Short-term prognosis after emergency department diagnosis of TIA. JAMA 2000;284:2901-6.

6 Burn J, Dennis M, Bamford R, Sandercock P, Wade D, Warlow C. Long-term risk of recurrent stroke after a first-ever stroke. The Oxfordshire Community Stroke Project. Stroke 1994;25:333-7.

7 Petty GW, Brown JR, Whisnant JP, Sicks JD, O'Fallon WM, Wiebers DO. Survival and recurrence after first cerebral infarctions. A population-based study in Rochester, Minnesota, 1975 through 1989. Neurology 1988;150:208-16.

8 Sacco RL, Shi T, Zamanillo MC, Kargman DE. Predictors of mortality and recurrence after hospitalized cerebral infarction in an urban community. The Northern Manhattan Stroke Study. Neurology 1994;44:626-34.

9 Ross R. Atherosclerosis - an inflammatory disease. N Engl J Med 1999;340:115-26.

10 Pietroiusti A, Diomedi M, Silvestrini M et al. CagA positive Helicobacter pylori strains are associated with atherosclerotic stroke. Circulation 2002;106:580-4.

11 Diomedi M, Pietroiusti A, Silvestrini M, Rizzato B, Cupini LM, Ferrante F, Magrini A, Bergamaschi A, Galante A, Bernardi G. CagA-positive Helicobacter pylori strains may influence the natural history of atherosclerotic stroke. Neurology 2004;63:800-4.

12 Corrado E, Rizzo M, Tantillo R, Muratori I, Bonura F, Vitale G, Novo S. Markers of inflammation and infection influence the outcome of patients with baseline asymptomatic carotid lesions: a 5-year follow-up study. Stroke 2006;37:482-6.

13 Gabrielli M, Santoliquido A, Cremonini F et al. CagA-positive cytotoxic $H$. pylori strains as a link between plaque instability and atherosclerotic stroke. Eur Heart J 2004;25:64-8.

14 Adams HP, Bendixen BH, Kappelle LJ, Biller J, Love BB, Gordon DL, Marsh EE III. Classification of subtype of acute ischaemic stroke: definition for use in a multicenter clinical trial: TOAST: Trial of Org 10172 in Acute Stroke treatment. Stroke 1993;24:35-41.

15 De Bray JM, Glatt B. Quantification of atheromatous stenosis in the extracranial internal carotid artery. Cerebrovasc Dis 1995;5:414-26.

16 Nicolaides AN, Shifrin EG, Bradbury A, Dhanjil S, Griffin M, Belcaro G, Williams M. Angiographic and duplex grading of internal carotid stenosis: can we overcome the confusion? $J$ Endovasc Surg 1996;3:158-65.

17 Bartels E. Vertebral sonography. In: Tagler CH, Babikian VL, Gomez CR, eds. Neurosonology. St. Louis: Mosby, 1995;83-100.

18 O'Leary DH, Polak JF, Kronmal RA, Manolio TA, Burke GL, Wolfson SK. Carotid-artery intima and media thickness as a risk factor for myocardial infarction and stroke in older adults. Cardiovascular HealthStudy Collaborative Research Group. N Engl J Med 1999;340:14-22.

19 Shine B, de Beer FC, Pepys MB. Solid phase radioimmunoassay for human C-reactive protein. Clin Chim Acta 1981;117:13-23.
20 Gill R, Kemp JA, Sabin C, Pepys MB. Human C-reactive protein increases cerebral infarct size after middle cerebral artery occlusion in adult rats. J Cereb Blood Flow Metab 2004;24:1214-8.

21 Smith CJ, Emsley HC, Vail A, Georgiou RF, Rothwell NJ, Tyrrell PJ, Hopkins SJ. Variability of the systemic acute phase response after ischemic stroke. J Neurol Sci 2006;251:77-81.

22 Whisnant JD, Busford JR, Bernstein EF, Cooper ES, Dyken ML, Eastone D. National Institute of Neurological Disorders and Stroke special report: classification of cerebrovascular disease. Stroke 1990;21:637-76.

23 Report of the WHO task force on stroke and other cerebrovascular disorders. Recommendations on stroke prevention, diagnosis and therapy. Stroke 1989;20:1407-31.

24 Peduzzi P, Concato J, Feinstein AR, Holford TR. Importance of events per independent variable in proportional hazards regression analysis. II. Accuracy and precision of regression estimates. J Clin Epidemiol 1995;48:1503-150.

25 Concato J, Peduzzi P, Holford TR, Feinstein AR. Importance of events per independent variable in proportional hazards analysis. I. Background, goals, and general strategy. J Clin Epidemiol 1995;48:1495-501.

26 Mayr M, Kiechl S, Mendall MA, Willeit J, Wick G, Xu Q. Increased risk of atherosclerosis is confined to CagA positive Helicobacter pylori strains. Prospective results from the Bruneck study. Stroke 2003;34:610-5.

27 Farsak B, Yildirir A, Akyon Y, Pinar A, Oc M, Boke E, Kes S, Tokgozoglu L. Detection of Chlamydia pneumoniae and Helicobacter pylori DNA in human atherosclerotic plaques by PCR. J Clin Microbiol 2000;38:4408-11.

28 Ameriso SF, Fridman EA, Leiguarda RC, Sevlever GE. Detection of Helicobacter pylori in human carotid atherosclerotic plaques. Stroke 2001;32:385-91.

29 Franceschi F, Sepulveda AR, Gasbarrini A, Pola P, Silveri NG, Gasbarrini G, Graham DY, Genta RM. Cross-reactivity between anti-CagA antibodies with vascular wall antigens. Possible pathogenic link between Helicobacter pylori infection and atherosclerosis. Circulation 2002;106:430-4.

30 Shinton R, Beevers G. Meta-analysis of relation between cigarette smoking and stroke. BMJ 1989;298:789-94.

31 Kool MJ, Hoeks AP, Struijker Boudier HA, Reneman RS, Van Bortel LMJ. Short- and long-term effects of smoking on arterial wall properties in habitual smokers. J Am Coll Cardiol 1993;22:1881-1886.

32 Yasue H, Hirai N, Mizuno Y, Harada E, Itoh T, Yoshimura M, Kugiyama K, Ogawa H. Low-grade inflammation, thrombogenicity, atherogenic lipid profile in cigarette smokers. Circ J 2006;70:8-13.

33 Grau G, Buggle F, Lichy C, Brandt T, Becher H, Rudi J. Helicobacter pylori infection ad an independent risk factor for cerebrovascular ischemia of atherotrombotic origin. J Neurol Sci 2001;186:1-5.

34 Preusch MR, Grau AJ, Buggle F, Lichy C, Bartel J, Black C, Rudi J. Association between cerebral ischemia and cytotoxin-associated gene-A-bearing strains of Helicobacter pylori. Stroke 2004;35:1800-4.

35 Bytzer B, O'Morain C. Treatment of Helicobacter pylori. Helicobacter 2005; 10:40-6.

36 Cutler AF, Vajravel M. Long-term follow-up of Helicobacter pylori serology after successful eradication. Am J Gastroenterol 1996;91:85-8. 